\title{
Dielectric Relaxations in Poly(ethylene oxide): Dependence on Molecular Weight
}

\author{
Kazunori SE, Keiichiro AdACHI, and Tadao KotAKA* \\ Department of Macromolecular Science, College of Science, \\ Osaka University, Toyonaka, Osaka 560, Japan.
}

(Received March 11, 1981)

\begin{abstract}
The dielectric properties of poly(ethylene oxide) (PEO) were examined for 10 samples ranging in molecular weight, $M$, from 190 to $2 \times 10^{6}$. Two dielectric relaxations were observed. The one observed at high temperature and low frequency is called $\alpha$ process, and the other at low temperature and high frequency is called $\beta$ one. The $\beta$ process is due to the local motion of amorphous PEO segments, and has a loss maximum temperature, $T_{\max }$, and an apparent activation energy, $E_{\mathrm{a}}$, independent of either $M$ or degree of crystallinity, $X_{\mathrm{c}}$. On the other hand, the $\alpha$ process, which is due to a large scale conformational rearrangement of amorphous PEO segments, is dependent on both $M$ and $X_{\mathrm{c}}$. The relaxation strength and $T_{\max }$ vary with $X_{\mathrm{c}}$, while the $E_{\mathrm{a}}$ and the relaxation width do not depend on $X_{\mathrm{c}}$ but exhibit an abrupt increase at $M$ of about $10^{4}$. These results may be due to change not only in $X_{\mathrm{c}}$ but also in the crystalline morphology with $M$.

KEY WORDS Poly(ethylene oxide) / Dielectric Relaxation /. Molecular Weight Dependence / Dielectric Loss Maximum Temperature / Relaxation Strength / Activation Energy / Relaxation Width / Degree of Crystallinity /
\end{abstract}

Polar amorphous polymers usually exhibit at least two dielectric absorption processes associated with the $\alpha$ and $\beta$ relaxations appearing in a hightemperature, low-frequency region and in a lowtemperature, high-frequency region, respectively. ${ }^{1,2}$ A number of dielectric studies on amorphous polymers were carried out for examining the influence of molecular weight, stereoregularity, chain topologies, and other external variables. ${ }^{1,2}$ Theoretical interpretations of these data were made to elucidate the molecular relaxation mechanisms underlying these dielectric absorption processes. ${ }^{1,3}$ As a result, the $\alpha$ relaxation was ascribed to the large-scale conformational rearrangements of the main chains, while the $\beta$ relaxation was ascribed to the motion of side groups and/or the small-scale "local mode" motion $^{3}$ of the main chains. Interestingly, not only the "local mode" $\beta$ relaxation but also the largescale $\alpha$ relaxation were found to be independent of molecular weight, except when the molecular weight was below $10^{4}$ or the polymer molecule possessed an effective dipole moment in the direction of the chain

* To whom correspondence should be addressed. contour. ${ }^{1}$

Similar data were also obtained for polar semicrystalline polymers such as polyethers which exhibited similar $\alpha$ and $\beta$ relaxations associated with the amorphous phase in the polymers. ${ }^{1-3}$ In certain cases, semicrystalline polymers exhibited a third relaxation, called the $\alpha_{c}$ relaxation $^{4}$ which is associated with the crystalline phase. ${ }^{1-4}$

In semicrystalline polymers, the degree of crystallinity and the crystalline morphology depend on molecular weight. ${ }^{5}$ In such polymers, the large-scale segmental motions in the amorphous phase may be influenced through constraints imposed by the crystalline region. ${ }^{3}$ Hence, the dielectric $\alpha$ relaxation may depend on the molecular weight through change not only in the degree of crystallinity but also in the crystalline morphologies. Thus, for poly(ethylene oxide), the crystalline morphology was reported to depend on the molecular weight in the following manner: samples of molecular weight between 1000 and 3500 always crystallized in a fully extended form from the melt, while samples with molecular weights of 10000 or above crystallized in folded chain crystals with the lamellar thickness, 
depending on the crystallization temperature. ${ }^{5}$

Many authors have studied the dielectric and mechanical relaxation behavior of poly(ethylene oxide) over wide ranges of temperature and frequency. ${ }^{1,2}$ Ishida et al. ${ }^{6}$ made the above-mentioned assignment of the molecular mechanisms to the $\alpha$ and $\beta$ processes while studying poly(ethylene oxide) single crystal mats. Koizumi and $\mathrm{Hanai}^{7}$ observed a relaxation process due to the space-charge polarization in poly(ethylene oxide) having a molecular weight below $10^{4}$. Connor et al. ${ }^{8}$ also made an extensive study of poly(ethylene oxide) samples having molecular weights between 200 and $2.8 \times 10^{6}$, and found that their dielectric properties depended on molecular weight as a result of the change in the degree of crystallinity. Arisawa et al. ${ }^{9}$ pointed out that identification of the $\alpha$ process was possible only by separating it from the contribution of direct-current conduction. Porter and Boyd ${ }^{10}$ examined crystalline and molten poly(ethylene oxide) in the $\mathrm{GHz}$ region, and concluded that the nature of segmental motions in the melt was essentially identical with the $\alpha$ relaxation in the solid state. They also pointed out that variation in crystallinity with molecular weight influenced the location of the $\alpha$ process in the frequency domain and the strength of relaxation. ${ }^{10}$

We also attempted to examine the dielectric behavior of poly(ethylene oxide) with molecular weight between 190 and $2 \times 10^{6}$ to elucidate the influences of molecular weight on the $\alpha$ and $\beta$ relaxations in greater detail. The reason for doing so was twofold: One was that few systematic studies have been available on the $\beta$ relaxation. The other was that a certain ambiguity has existed in the available data for the $\alpha$ process of this polymer. Since poly(ethylene oxide) is a polar and water soluble polymer, the $\alpha$ process is usually masked by a large contribution of an ionic direct-current conduction at high temperatures and low frequencies, and cannot be readily measured. ${ }^{9}$ It was thus necessary to remove moisture and ionic contaminants as thoroughly as possible before carrying out dielectric measurements. The results of these studies are described in this article.

\section{EXPERIMENTAL}

\section{Materials}

Poly(ethylene oxide) (PEO) samples of molecular weight, $M$, below $2 \times 10^{4}$ were purchased from Wako Pure Chemicals Co., while those with $M$ above $2 \times 10^{5}$ were Polyox samples (Union Carbide Corp.) obtained through the courtesy of Professor Guy C. Berry, Carnegie-Mellon University. Two more samples with $M=3.1 \times 10^{4}$ and $1.60 \times 10^{5}$ were prepared in this laboratory by polymerizing ethylene oxide monomers with strontium carbonate as the catalyst. ${ }^{11}$ All the samples were purified by repeated dissolution-reprecipitation a few times with a toluene/methanol mixture as the solvent and $n$-hexane as the precipitant.

\section{Methods}

Average molecular weights and heterogeneities were estimated by intrinsic viscosity measurements in $20^{\circ} \mathrm{C}$ benzene ${ }^{12}$ (for low $M$ samples) or in $30^{\circ} \mathrm{C}$ water ${ }^{13}$ (for high $M$ samples) and by gel permeation chromatography (GPC: Model HLC-801A, Toyo Soda Mgf. Co.) using a low-angle laser-light scattering (LALLS) monitor (Model LS-8, Toyo Soda Mfg. Co.) with tetrahydrofuran as the carrier. The degree of crystallinity, $X_{\mathrm{c}}$, was determined by density measurement with a Welde-type pycnometer or by differential scanning calorimetry (DSC: Model 8055, Rigaku Denki Co.). Before making DSC measurements, the PEO samples were annealed under the same conditions as for the samples on which dielectric measurements were made. The results of sample characterization are summarized in Table I.

The dielectric measurements were made in the range from $3 \mathrm{~Hz}$ to $1 \mathrm{MHz}$ on a Cole-Gross type capacitance bridge (Showa Denki), a capacitance bridge (General Radio, Model 1615A), and an automatic capacitance bridge (Yokogawa-HewlettPackard, Model YHP 4070A). In making a measurement, a PEO specimen was inserted in an improvised three-electrode cell for liquid samples with an air-tight brass container. ${ }^{14}$ The sample was degassed and melted in vacuum by heating to $100^{\circ} \mathrm{C}$, so as to achieve good contact between the specimen and electrode surfaces. In routine measurements, the specimen was further dried at $100^{\circ} \mathrm{C}$ in vacuum of $10^{-4}$ torr for about $100 \mathrm{~h}$ to completely remove any moisture. The container was then filled with helium, and rapidly cooled (at about $4^{\circ} \mathrm{C} / \mathrm{min}$ rate) to room temperature. In order to avoid any possible ionic conduction that would make the $\alpha$ relaxation of PEO difficult to observe, 
Dielectric Relaxations in Poly(ethylene oxide)

Table I. Characteristics of PEO samples

\begin{tabular}{|c|c|c|c|c|c|}
\hline \multicolumn{2}{|c|}{ Sample code } & \multicolumn{2}{|c|}{ Commercial name } & \multirow{2}{*}{$\frac{M_{v}^{\mathrm{a}}}{1.9 \times 10^{2}}$} & \multirow[t]{2}{*}{$X_{\mathrm{c}}^{\mathrm{b}}$} \\
\hline PEO & 190 & PEG & 200 & & \\
\hline PEO & 300 & PEG & 400 & $3.0 \times 10^{2}$ & \\
\hline PEO & 850 & PEG & 1000 & $8.5 \times 10^{2}$ & 78 \\
\hline PEO & 6500 & PEG & 6500 & $6.5 \times 10^{3}$ & 94 \\
\hline PEO & 20000 & PEG & 20000 & $2.0 \times 10^{4}$ & 85 \\
\hline Polym & 3.1 & \multicolumn{2}{|l|}{$-^{c}$} & $3.1 \times 10^{4}$ & 85 \\
\hline Polym & 16 & \multicolumn{2}{|l|}{$-^{c}$} & $1.6 \times 10^{5}$ & 83 \\
\hline Polyox & 20 & Polyox WSRN- & 85 & $2 \times 10^{5}$ & 74 \\
\hline Polyox & 90 & Polyox WSR- & 1105 & $9 \times 10^{5}$ & 74 \\
\hline Polyox & 200 & Polyox WSR- & 301 & $2 \times 10^{6}$ & 75 \\
\hline
\end{tabular}

a $M_{v}=$ Viscosity average molecular weight.

b $X_{\mathrm{c}}=$ Crystallinity $\%$ determined by DSC.

c Prepared with $\mathrm{SrCO}_{3}$ catalyst.

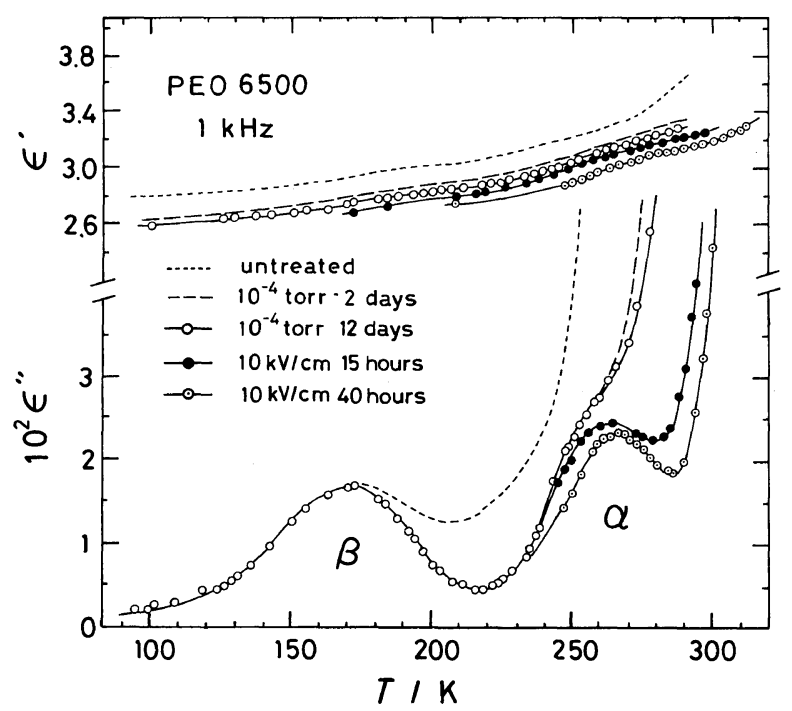

Figure 1. Effect of purifying treatments, drying and purging, on temperature dependence of dielectric constant $\varepsilon^{\prime}$ and loss $\varepsilon^{\prime \prime}$ for a PEO 6500 specimen.

we employed a method introduced by Osaki et al., ${ }^{15}$ who eliminated ionic contaminants by applying an electrostatic field of about $10 \mathrm{kV} \mathrm{cm}^{-1}$ across the specimen. After this treatment, we carried out dielectric measurements on the samples.

\section{RESULTS}

\section{Effects of Drying and Purging}

Figure 1 shows the temperature dependence of dielectric loss, $\varepsilon^{\prime \prime}$, at $100 \mathrm{~Hz}$ for a variously treated sample with $M=6500$, having the highest degree of crystallinity $X_{\mathrm{c}}(\cong 94 \%)$ for the samples examined here. The untreated specimen exhibited a steep rise in $\varepsilon^{\prime \prime}$ in the 220 to $240 \mathrm{~K}$ region, and the $\alpha$ process is completely buried below this rise. After drying at $100^{\circ} \mathrm{C}$ under $10^{-4}$ torr for two days, the $\alpha$ process could barely be observed as a shoulder of the steeply increasing $\varepsilon^{\prime \prime}$ curve at about $255 \mathrm{~K}$. Further drying for 10 more days did not improve the results.

Hence, we treated the dried specimen by applying the static field of $10 \mathrm{kV} \mathrm{cm}^{-1}$ for $40 \mathrm{~h}$. The $\alpha$ peak 


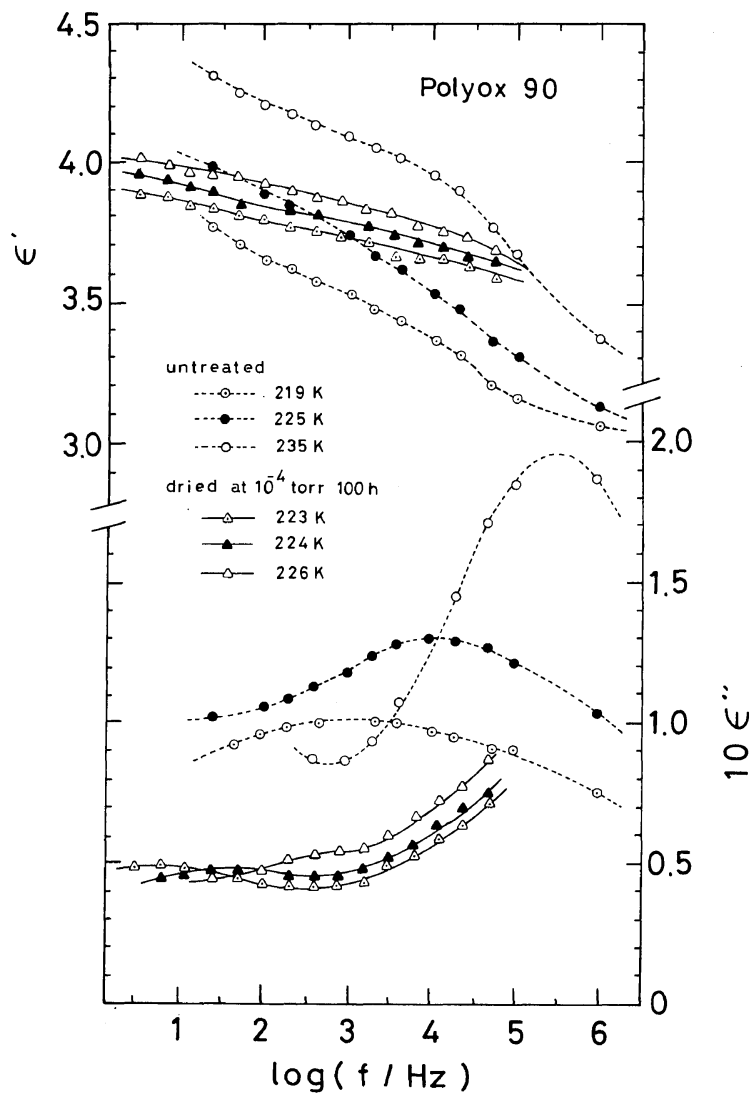

Figure 2. Effect of drying (at $100^{\circ} \mathrm{C}$ under $10^{-4}$ torr for $100 \mathrm{~h}$ ) on frequency dispersion curves of $\varepsilon^{\prime}$ and $\varepsilon^{\prime \prime}$ for a polyox 90 specimen.

finally appeared around $260 \mathrm{~K}$. For an untreated Polyox 200 specimen, we found a similar steep rise in $\varepsilon^{\prime \prime}$ in the range from about 200 to $230 \mathrm{~K}$, while after drying the specimen for 7 days, the $\alpha$ peak was clearly observed in the range 230 to $260 \mathrm{~K}$.

Similar effects from drying and purging were also observed in the frequency dependence of $\varepsilon^{\prime \prime}$. Figure 2 compares the $\varepsilon^{\prime \prime}$ curves in the $\alpha$ relaxation region for an untreated and a dried-and-purged Polyox 90 specimen. We see that the magnitude of $\varepsilon^{\prime \prime}$ for the purified specimen is apparently significantly lower. In this $\varepsilon^{\prime \prime}$ vs. $\log f$ curve, we can recognize two relaxation processes at about $10 \mathrm{~Hz}$ and above $100 \mathrm{kHz}$. Extrapolation of the Arrhenius plot obtained from the data shown in Figure 5 indicates that the $\beta$ peak is situated about $300 \mathrm{kHz}$ at $224 \mathrm{~K}$. Therefore, we may conclude that the increase in $\varepsilon^{\prime \prime}$ above $100 \mathrm{kHz}$ is due to the $\beta$ process and that the small peak at about $10 \mathrm{~Hz}$ is due to the $\alpha$ process.
These results indicate that impurities cause a drastic change in the dielectric spectrum, so that drying and purging are necessary to obtain reliable data on dielectric relaxations in highly hydrophilic polymers such as PEO. For this reason, our later measurements were made on the specimens dried at $100^{\circ} \mathrm{C}$ under $10^{-4}$ torr for $100 \mathrm{~h}$, and purged of ionic impurities overnight at a static field of $10 \mathrm{kV} \cdot \mathrm{cm}^{-1}$.

\section{Magnitude of Dielectric Relaxation}

Figures 3, 4, and 5 show the temperature dependence of the dielectric constant, $\varepsilon^{\prime}$ and loss, $\varepsilon^{\prime \prime}$ for PEO samples with $M=190,850$, and $9 \times 10^{5}$, respectively. In Figure 6, the temperature dependence curves obtained at $1 \mathrm{kHz}$ are compared for four purified samples with $M$ ranging from 300 to $1.6 \times 10^{5}$. These $\varepsilon^{\prime \prime}$ curves clearly exhibit two peaks at about 240 and $160 \mathrm{~K}$, corresponding respectively to the $\alpha$ and $\beta$ relaxations. The heights of these $\alpha$ 
peaks are several times smaller than those reported previously for corresponding PEO samples. ${ }^{8}$

The maginitude of the dielectric relaxation or the relaxation strength, $\Delta \varepsilon$, for the $\beta$ process was determined by standard methods. ${ }^{1}$ However, an accurate estimation of $\Delta \varepsilon$ for the $\alpha$ process by the standard method such as the Cole-Cole plot ${ }^{16}$ was difficult, since the $\alpha$ process was usually obscured by the preceding low-temperature, high-frequency process followed by the contribution of directcurrent conduction in the high temperature, lowfrequency region. Thus, in most cases, the values of $\Delta \varepsilon$ for the $\alpha$ process were determined simply by

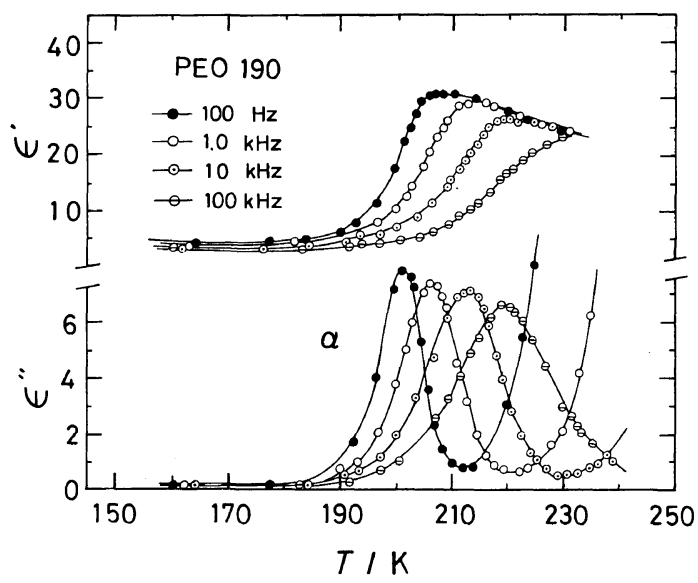

Figure 3. Temperature dependence of $\varepsilon^{\prime}$ and $\varepsilon^{\prime \prime}$ for PEO 190 sample. the difference in dielectric constants at the high and low temperature sides of the loss peak.

In Figure 7, the values of $T \Delta \varepsilon$ for the $\alpha$ and $\beta$ processes are plotted against the degree of crystallinity, $X_{\mathrm{c}}$. The $T \Delta \varepsilon$ for the $\alpha$ process decreases almost linearly with increasing $X_{\mathrm{c}}$, although the points are somewhat scattered, and was extrapolated to zero at the $100 \%$ crystallinity. For PEO with $M=2.8 \times 10^{6}$ and $X_{\mathrm{c}}=61 \%$, Connor et al. ${ }^{8}$ reported the value of $T \Delta \varepsilon$ to be 347 , while in our results, an untreated sample with the corresponding $M$ and $X_{\mathrm{c}}$ gave a similar magnitude of $T \Delta \varepsilon$, but the treated sample gave a $T \Delta \varepsilon$ as small as 140 .

The value of $T \Delta \varepsilon$ for the $\beta$ process also decreases with increasing $X_{\mathrm{c}}$, but does not approach zero at $100 \%$ crystallinity. This is in agreement with the fact that both the $\alpha$ and $\beta$ processes are related to the molecular motion in the amorphous region, and also that the $\beta$ process is related to motion in the crystalline defects, ${ }^{6}$ which can hardly be identified as the amorphous fraction by the DSC method employed in the present study.

From the data given in Figure 7, the value of $T \Delta \varepsilon$ for the $\alpha$ relaxation per unit volume of PEO amorphous phase was estimated to be about 380 . We then estimated the root mean-square effective dipole moment, $\left\langle\mu^{2}\right\rangle^{1 / 2}$, per ethylene oxide unit involved in the $\alpha$ process to be $0.617 \mathrm{D}$ using the Onsager equation,,$^{17,1,2}$

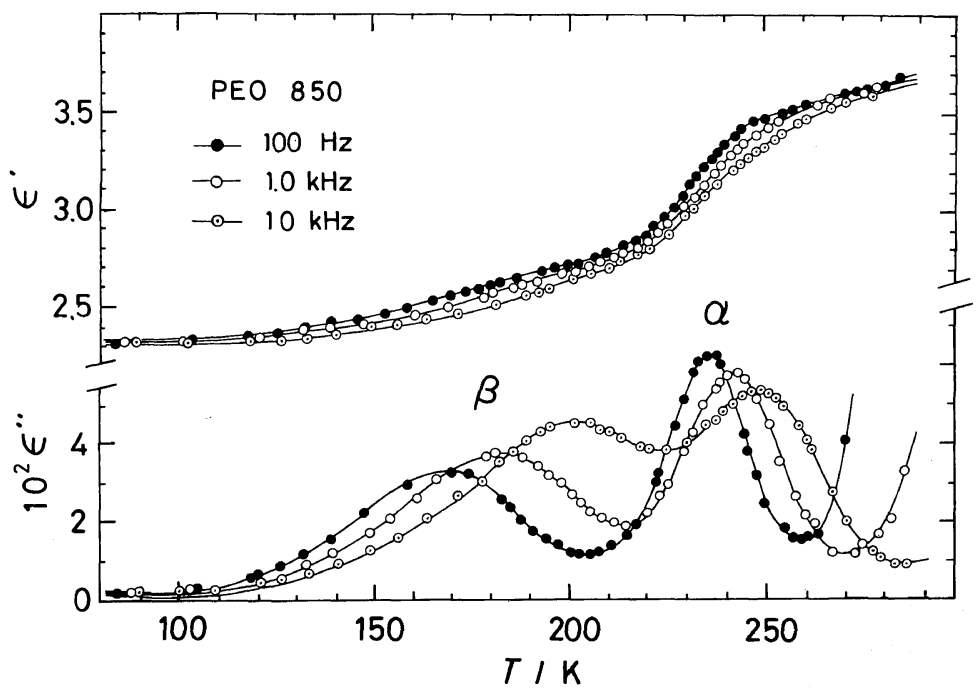

Figure 4. Temperature dependence of $\varepsilon^{\prime}$ and $\varepsilon^{\prime \prime}$ for PEO 850 sample. 


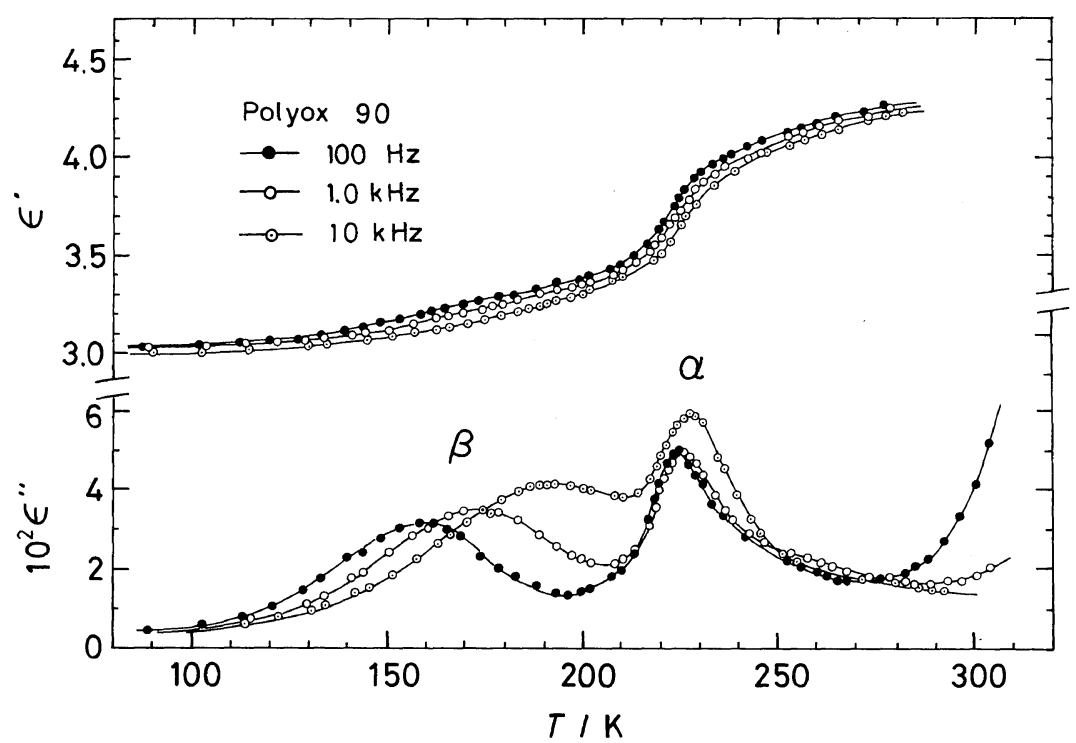

Figure 5. Temperature dependence of $\varepsilon^{\prime}$ and $\varepsilon^{\prime \prime}$ for Polyox 90 sample.

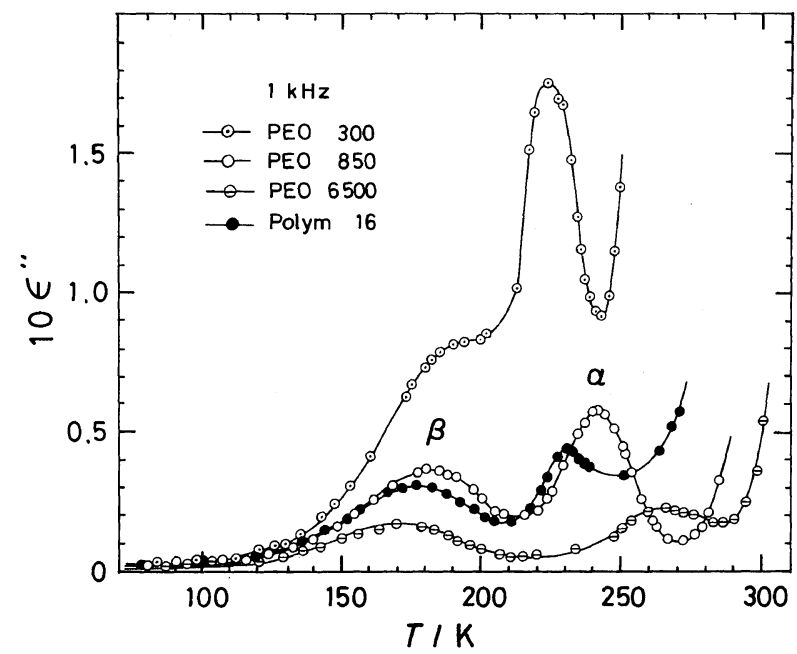

Figure 6. Comparison of temperature dependence curves of $\varepsilon^{\prime \prime}$ determined at $1 \mathrm{kHz}$ frequency for four PEO samples.

$$
\Delta \varepsilon=\frac{4 \pi N\left\langle\mu^{2}\right\rangle}{3 k T} \frac{3 \varepsilon^{\prime}(0)}{2 \varepsilon^{\prime}(0)+\varepsilon^{\prime}(\infty)}\left[\frac{\varepsilon^{\prime}(\infty)+2}{3}\right]^{2}
$$

Here $N$ is the average dipole number (ethylene oxide units) per unit volume.

The mean-square dipole moment ratio, $\left\langle\mu^{2}\right\rangle / \mathrm{nm}^{2}$, is 0.19 , where $n m^{2}$ represents the sum of the squares of the individual dipole moments in the molecule. $^{18,19}$ This value is only about one third that reported by Flory, ${ }^{18}$ who used the rotational isomeric state theory and data for PEO oligomers in solution. ${ }^{18-22}$ The conformational restriction is obviously larger for PEO segments in the solid state than for those in solution and the gaseous state.

Loss Maximum Temperature and Activation Energy

Plots of $\log$ (frequency $f / \mathrm{Hz}$ ) versus the reciprocal of the loss maximum temperature, $T_{\max }$, are often 


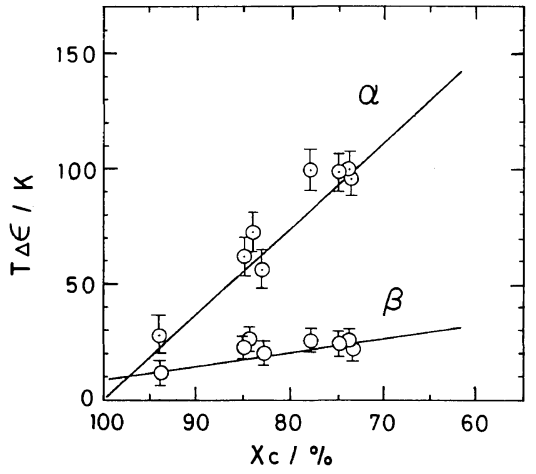

Figure 7. Plots of dielectric relaxation strength $T \Delta \varepsilon$ versus degree of crystallinity $X_{\mathrm{c}}$ for the $\alpha$ and $\beta$ processes.

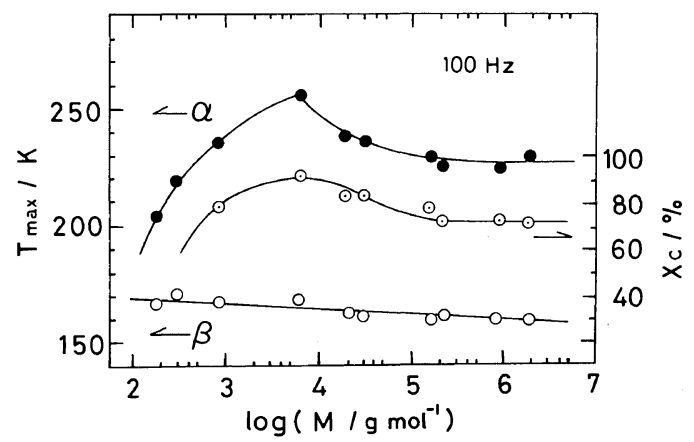

Figure 8. Plots of $\log M$ versus degree of crystallinity $X_{\mathrm{c}}$ and dielectric loss maximum temperatures $T_{\max }$ determined at $100 \mathrm{~Hz}$ frequency for the $\alpha$ and $\beta$ processes.

referred to as the activation plot or the dispersion map. ${ }^{23}$ The activation plots for the $\alpha$ process are of the Williams-Landel-Ferry (WLF) type, and the activation energy, $E_{\mathrm{a}}$, depends on temperature or frequency. The plot shifts to higher temperature with increasing $M$ from 190 to 6500 . However, the plot shifts back to lower temperature, and the slope becomes much steeper, as $M$ further increases above $10^{4}$. On the other hand, the activation plots for the $\beta$ process are all of the Arrhenius type, with the slope and location being independent of $M$.

In Figure 8, the degree of crystallinity, $X_{\mathrm{c}}$, and the loss maximum temperatures, $T_{\max }$, at $100 \mathrm{~Hz}$ are plotted against $\log M$. The $T_{\max }$ for the $\alpha$ process has a maximum for the sample with $M=6500$; this sample incidentally has the largest $X_{\mathrm{c}}$ among the samples examined here. The $T_{\max }^{\alpha}$ varies roughly with $X_{\mathrm{c}}$. On the other hand, the $T_{\max }$ for the $\beta$ process is nearly independent of either $X_{\mathrm{c}}$ or $M$.

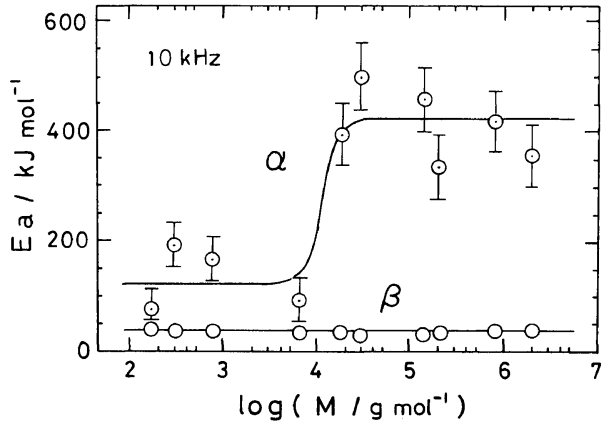

Figure 9. Plots of $\log M$ versus apparent activation energy $E_{\mathrm{a}}$ determined at $10 \mathrm{kHz}$ for the $\alpha$ and $\beta$ processes.

In Figure 9, the apparent activation energies at $10 \mathrm{kHz}$ for the $\alpha$ and $\beta$ processes are plotted against $\log M$. The values $E_{\mathrm{a}}$ for the $\alpha$ process are about $120 \mathrm{~kJ} \mathrm{~mol}^{-1}$ for samples with $M$ below 6500 , and sharply increase to about $420 \mathrm{~kJ} \mathrm{~mol}^{-1}$ for samples with $M$ above $10^{4}$. Connors et al. ${ }^{8}$ reported that the values of $E_{\mathrm{a}}{ }^{\alpha}$ for samples with $M$ from $3 \times 10^{4}$ to $2.8 \times 10^{6}$ were about $230 \mathrm{~kJ} \mathrm{~mol}^{-1}$. Ishida et al. ${ }^{6}$ reported for Polyox 200 an $E_{\mathrm{a}}^{\alpha}$ value of about $200 \mathrm{~kJ} \mathrm{~mol}^{-1}$. These values are nearly the same for untreated samples but only about one half as much as the values for the dried-and-purged samples. The value of $E_{\mathrm{a}}$ for the $\beta$ process, however, is about $40 \mathrm{~kJ} \mathrm{~mol}^{-1}$, and is independent of either $X_{\mathrm{c}}$ or $M$.

\section{Relaxation Width}

Even in the carefully treated PEO samples, the frequency dependence of $\varepsilon^{\prime \prime}$ for the $\alpha$ process was obscured by the direct-current conduction and the high-frequency $\beta$ process. The dispersion curves were thus determined only over a limited range of frequency for a few samples. Figure 10 shows the reduced plots, $\varepsilon^{\prime \prime} / \varepsilon_{\max }^{\prime \prime}$ versus $\log \left(f / f_{\max }\right)$, for the $\alpha$. and $\beta$ processes. Apparently, the lowest $M$ PEO sample has a much narrower $\alpha$ curve than the higher $M$ samples, implying that the former has a much narrower distribution of dielectric relaxation time. The reduced curves for the $\beta$ process are nearly the same for all samples examined here.

\section{DISCUSSION}

The present data indicate that the $T_{\max }$ and $E_{\mathrm{a}}$ of the $\beta$ relaxation process are essentially independent 


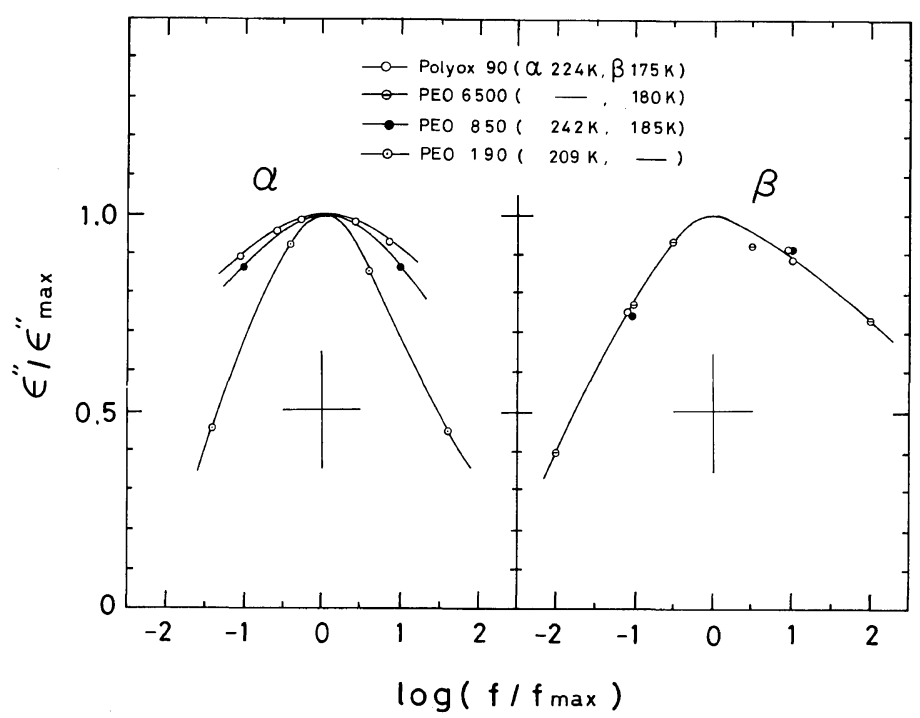

Figure 10. Plots of $\varepsilon^{\prime \prime} / \varepsilon_{\max }^{\prime \prime}$ versus $\log \left(f / f_{\max }\right)$ for the $\alpha$ and $\beta$ processes for four PEO samples.

of either $X_{\mathrm{c}}$ or $M$. This is in agreement with the previous conclusion ${ }^{3,6}$ that the $\beta$ process is due to the local motion of the PEO segments. Our recent studies on PEO-bisphenol A polycarbonate (PC) multiblock copolymers revealed that the $\beta$ relaxation seems to involve the motion of PEO segments as small as a dimer or a sequence of five or six bonds. ${ }^{26}$ We concluded from the relaxation strength of the $\beta$ relaxation of these copolymers that the underlying molecular mechanism is presumably the local $t g^{+} t \rightleftarrows t g^{-} t$ transition in the PEO segments. ${ }^{26}$

For the $\alpha$ relaxation process, we obtained somewhat different results from those of other workers, ${ }^{6,8,10}$ especially for samples with high $M$ : The values of $T \Delta \varepsilon$ are smaller, and the values of $E_{\mathrm{a}}{ }^{\alpha}$ are larger than the reported values. ${ }^{6,8}$ These discrepancies appear to be due to the difference in moisture and/or impurity content.

The $\alpha$ relaxation mechanism is undoubtedly the largescale conformational rearrangement in the amorphous regions, subject to constraints from the crystalline regions. The most interesting point is that the loss maximum temperature, $T_{\mathrm{m}}{ }^{\alpha}$, varies roughly with the degree of crystallinity, $X_{\mathrm{c}}$, while the activation energy, $E_{\mathrm{a}}{ }^{\alpha}$, does not depend on $X_{\mathrm{c}}$ but exhibits a rather abrupt increase at $M$ above $10^{4}$. The relaxation width is larger for high $M$ samples than that for low $M$ samples. This implies that the $\alpha$ relaxation process is influenced not only by variation in $X_{\mathrm{c}}$, but also by other factors such as crystalline morphology. According to Arlie et al., ${ }^{5}$ PEO samples with $M$ below 3500 form extended chain crystals when quenched from the melt. On the other hand, samples with $M$ above $10^{4}$ form folded chain lamellae of about $200 \AA$ in thickness. Tadokoro et al. reported that in such lamellar crystallites, the PEO molecule assumes a $7 / 2$ helical conformation, ${ }^{27}$ meaning that each lamella contains sequences of about 3500 in $M$. Among the PEO samples examined here, the sample with $M=6500$ had the narrowest distribution of molecular weight and the highest degree of $X_{\mathrm{c}}$. In its crystallites, each molecule presumably assumes a hair-pin conformation with one tight fold. On the other hand, PEO molecules with $M=10^{4}$ barely form folded chain lamellae with one loose fold or, at most, two tight folds. Therefore, in PEO samples with $M$ below $10^{4}$, the amorphous region consists of short free chains and/or cilia with one end fixed to extended chain crystallites. In samples with $M$ above $10^{4}$, the amorphous region consists of cilia, loops of a switchboard type,$^{28}$ and interlamellar bridges. For samples with larger $M$, the extent of the latter two morphologies becomes greater. The large-scale motion of the PEO segments in the loops and bridges, in which both ends of each chain are fixed to crystallites, should be more restricted and have a greater $E^{\alpha}$ value than that of the cilia. PEO samples with larger $M$ containing crystallites of different size should also have a broader relaxation time distri- 
bution. These differences in the degree of crystallinity $X_{\mathrm{c}}$ as well as in the crystalline morphology explain the features of the $\alpha$ relaxation process described above for PEO samples of different molecular weight.

\section{REFERENCES}

1. N. G. McCrum, B. E. Read, and G. Williams, "Anelastic and Dielectric Effects in Polymeric Solids," John Wiley \& Sons, Ltd., New York, 1967.

2. P. Hedvig, "Dielectric Spectroscopy of Polymers," Adam Hilger Ltd., Bristol, 1977.

3. Y. Ishida, J. Polym. Sci., A-2, 7, 1835 (1969).

4. M. Takayanagi, "Proceedings of the Fourth International Congress on Rheology," Vol. 1, E. H. Lee, Ed., Providence, Rhode Island, 1963, p 161.

5. J. P. Arlie, P. A. Spegt, and A. E. Skoulios, Makromol. Chem., 99, 170 (1966); ibid., 104, 212 (1967); B. Wunderlich, "Macromolecular Physics," Vol. 1, Academic Press, Inc., New York, 1973, pp 199-200.

6. Y. Ishida, M. Matsuo, and M. Takayanagi, J. Polym. Sci., B, 3, 321 (1965).

7. N. Koizumi and T. Hanai, J. Phys. Chem., 60, 1496 (1956).

8. T. M. Connor, B. E. Read, and G. Williams, J. Appl. Chem., 14, 74 (1964).

9. K. Arisawa, K. Tsuge, and Y. Wada, Jpn. J. Appl. Phys., 4, 138 (1965).

10. C. H. Porter and R. H. Boyd, Macromolecules, 4, 589 (1971).

11. F. N. Hill, F. E. Bailey, Jr., and J. T. Fits, Ind. Eng. Chem., 50, 5 (1958).

12. C: Sadron and P. Rempp, J. Polym. Sci., 29, 127
(1958).

13. F. E. Bailey, Jr., J. L. Kucera, and L. G. Imhof, $J$. Polym. Sci., 32, 517 (1958).

14. K. Adachi, Y. Hirose, and Y. Ishida, J. Polym. Sci., Polym. Phys. Ed., 13, 737 (1975).

15. S. Osaki, S. Uemura, and Y. Ishida, J. Polym. Sci., $A-2,9,585$ (1971).

16. K. S. Cole and R. H. Cole, J. Chem. Phys., 9, 314 (1941).

17. H. Froelich, "Theory of Dielectrics," Oxford Univ. Press, London, 1958.

18. P. J. Flory, "Statistical Mechanics of Chain Molecules," John Wiley, New York, 1969, pp 165172.

19. T. Uchida, Y. Kurita, N. Koizumi, and M. Kubo, $J$. Polym. Sci., 21, 313 (1956).

20. J. Marchal and H. Benoit, J. Polym. Sci., 23, 223 (1957).

21. A. Kotera, K. Suzuki, K. Matsumura, T. Nakano, T. Oyama, and U. Kambayashi, Bull. Chem. Soc., Jpn., 35, 797 (1962).

22. J. Bak, G. Elefante, and J. E. Mark, J. Phys. Chem., 71, 4007 (1967).

23. Y. Wada, "Kobunshi no Kotaibussei," Baifukan, Tokyo, 1971.

24. M. L. Williams, R. F. Landel, and J. D. Ferry, J. Am. Chem. Soc., 77, 3101 (1955).

25. J. D. Ferry, "Viscoelastic Properties of Polymers," 3rd ed, John Wiley, New York, 1980, Chapter 11.

26. T. Suzuki and T. Kotaka, Macromolecules, 13, 1495 (1980).

27. H. Tadokoro, Y. Chatani, Y. Yoshihara, S. Tahara, and S. Murahashi, Makromol. Chem., 23, 109 (1964).

28. See, for example, B. Wunderlich, Macromolecular Physics, Vol. 1, Academic Press, New York, 1973, pp $206-208$. 